\title{
O jardim de fungo atua como um molde para a construção das câmaras em formigas cortadeiras?
}

\author{
Fungus garden acts as a template for the construction of chambers in ants?
}

\author{
Roberto da Silva Camargo ${ }^{I^{*}}$ Juliane F. S. Lopes $^{\mathrm{I}}$ Luiz Carlos Forti ${ }^{\mathrm{II}}$
}

\section{RESUMO}

\begin{abstract}
Os ninhos adultos das formigas cortadeiras (gênero Atta e Acromyrmex) são compostos de milhares de câmaras subterrâneas, as quais abrigam o jardim de fungo, lixo e a população desses insetos. Entretanto, como as câmaras são construídas? Para responder essa questão, nós hipotetizamos que o jardim de fungo atua como um molde para a construção das câmaras. Assim, foram utilizadas 20 colônias de 6 meses de idade, divididas em quatro séries experimentais: padrão (quantidade normal de jardim de fungo); metade de jardim de fungo; dobro de jardim de fungo e sem jardim de fungo (Testemunha). As variáveis estudadas foram: parâmetros morfológicos das estruturas (túneis e câmara formada); fluxo das atividades das operárias; volume de solo escavado. Como se esperava, o jardim de fungo atua como um molde para a construção das câmaras em formigas cortadeiras. Os resultados foram: o tratamento sem jardim de fungo não apresentou nenhuma câmara, apenas túneis, em contraposição às demais séries experimentais que apresentaram no mínimo 2 câmaras, com dimensões similares; $o$ fluxo das operárias carregando pellet de solo por minuto durante as 72 horas diferiu estatisticamente entre as séries experimentais $e$, finalmente, o volume do solo escavado foi resultado da taxa de escavação das operárias, diferindo estatisticamente entre as séries experimentais. Os resultados corroboram a hipótese de que o jardim de fungo atua como um molde para a construção da câmara. A ausência de uma estrutura funcional como uma câmara, quando o jardim de fungo está ausente, comprova a hipótese.
\end{abstract}

Palavras-chave: formigas cortadeiras, escavação do ninho, divisão de trabalho.

\section{ABSTRACT}

Adult nests of leaf cutting ants (genus Atta and Acromyrmex) are composed of thousands of underground chambers, which harbor the fungus garden, garbage and their population. However, how the chambers are constructed? To answer this question, we hypothesized that the fungus garden acts as a template for the chambers construction. Thus, we used 20 colonies of 6 months of age, divided into four treatments: Normal (Control); Half fungal symbiont; Double symbiont fungus and No symbiotic fungus. The variables studied were: morphology, (tunnels and chamber formed); flow of activities of workers and volume of soil excavated. As expected, treatment no symbiotic fungus did not have any cameras, just tunnels, as opposed to other treatments that showed at least two chambers, with similar dimensions. The flow of workers carrying pellet of soil per minute for 72 hours, it differed between treatments. Thus, the volume of excavated soil was the result of the excavation rate of workers, differences among the treatments. The results confirm the hypothesis that the symbiotic fungus acts as a template for the construction of the chamber. The absence of a functional structure as a chamber when is absent of symbiont fungus proves the hypothesis.

Key words: leaf-cutting ants, digging the nest, division of labor.

\section{INTRODUÇÃO}

Os ninhos adultos das formigas cortadeiras (gênero Atta) são conhecidos por serem grandes e complexos, composto de milhares de câmaras subterrâneas, as quais abrigam o jardim de fungo, lixo e a população desses insetos (MOREIRA et al., 2004, HÖLLDOBLER \& WILSON, 2009). Mas a criação dessas estruturas é coordenada coletivamente por simples atos comportamentais de escavação das operárias, ou seja, a simples retirada de solo com as mandíbulas (CASSIL et al., 2002).

${ }^{I}$ Mirmecolab, Universidade Federal de Juiz de Fora (UFJF), Instituto de Ciências Biológicas. Rua José Lourenço Kelmer, s/n, Campus Universitário, Bairro São Pedro, 36036-900, Juiz de Fora, MG, Brasil. E-mail: camargobotucatu@ yahoo.com.br. *Autor para correspondência.

"Laboratório de Insetos Sociais-Praga, Departamento de Produção Vegetal, Faculdade de Ciências Agronômicas, Universidade Estadual paulista (UNESP), Botucatu, SP, Brasil. 
Experimentalmente, comprovou-se que muitas espécies de formigas utilizam gradientes de umidade e temperatura para construir seus ninhos e distribuir espacialmente ovos, larvas e pupas (BRIAN, 1983; CEUSTERS, 1986). O comportamento de escavação também é influenciado por heterogeneidades no ambiente, elas tendem a caminhar, construir, armazenar e por ovos ao longo dessas heterogeneidades (THERAULAZ et al., 1999). A formiga cortadeira Atta sexdens, o qual o surgimento do ninho se inicia com a fêmea recémacasalada (rainha), quando desce ao solo, destaca suas asas e, em seguida, escava durante horas o seu próprio ninho. Este ninho consiste de um túnel vertical, cerca de 8,5 a $15 \mathrm{~cm}$ de comprimento e diâmetro de 9 a $12 \mathrm{~mm}$, e de uma pequena câmara, com dimensões de 2,2 a $2,5 \mathrm{~cm}$ de altura e 3 a $4,5 \mathrm{~mm}$ de diâmetro da base. O túnel é obstruído com parte do solo removido durante a ampliação da câmara inicial, no sentido superfície do solo - câmara. A rainha fica enclausurada, e expele uma pequena porção do jardim de fungo trazida por ela em sua cavidade infra-bucal (AUTUORI, 1942). Quando as primeiras operárias emergem, estas reabrem o canal que foi obliterado pela rainha, de forma que permita o fluxo de operárias durante as atividades de forrageamento, remoção de solo e refugos do interior do ninho.

Mas como as operárias constroem essa nova câmara? Sabe-se que os animais utilizam molde para a construção de ninhos. Estes são guiados por esse molde do que irão construir, sendo já presente no ambiente sob a forma de pistas físicas e químicas (THERAULAZ, et al. 1999). Por exemplo, alguns pássaros utilizam as medidas corporais como um molde para a confecção de seu ninho (HANSELL, 2007). Analogamente, será que as operárias de formigas cortadeiras utilizam o jardim de fungo para a construção de novas câmaras? Dessa forma, nós hipotetizamos que o jardim de fungo atua como um molde tridimensional para a construção das câmaras.

\section{MATERIAL E MÉTODOS}

\section{Colônias estudadas}

Foram utilizadas 20 colônias de Atta sexdens rubropilosa, de 6 meses de idade, mantidas em laboratório desde a revoada da rainha em 2011, que ocorreu na cidade de Juiz de Fora, MG.

\section{Caixas de observação}

Foram utilizadas 4 caixas de vidro com dimensões de: $30 \mathrm{~cm}$ de comprimento e altura com $4 \mathrm{~cm}$ de largura. Estas foram preenchidas com solo
(Latossolo, densidade do solo $=1,6 \mathrm{~g} / \mathrm{cm}^{3}$; conteúdo de água: $5,4 \%$ ) coletado a $60 \mathrm{~cm}$ de profundidade e peneirado. Nesse aparato, foi construído manualmente (artificial) uma pequena câmara a $15 \mathrm{~cm}$ de profundidade da superfície do solo com dimensões de 2,5-3cm de comprimento e largura, com $1,5 \mathrm{~cm}$ de altura, cuja função foi estimular as operárias a escavar. Acima desse aparato, foi colocada uma pequena caixa de vidro $(10 \times 8 \times 5 \mathrm{~cm})$, para receber os tratamentos das diferentes situações experimentais (Figura 1).

Cinco colônias foram utilizadas para se ter uma média do número médio de operárias pequenas e médias, bem como, o volume de jardim de fungo cultivado. Os ninhos continham em média 130,8 $\pm 17,3$ operárias médias (largura da cabeça de 1,0 a 2,5mm), $163,2 \pm 10,5$ pequenas (largura da cabeça de 0,7 a $0,9 \mathrm{~mm})$, e $14,5 \pm 3,2 \mathrm{~g}$ de jardim de fungo.

\section{Escavação pelas operárias}

Foi avaliada a taxa de escavação das operárias frente a diferentes situações experimentais, sendo composto de 4 séries experimentais com 5 repetições (5 colônias):

Tratamento 1 - Padrão: número fixo de operárias (foi a média da população de ninhos de 6 meses), com o peso médio do jardim de fungo $(14,5 \pm 3,2 \mathrm{~g})$ encontrado em colônias de 6 meses $(\mathrm{N}=5)$. Essa idade foi escolhida, pois sabe-se que, em ambiente natural, ocorre a construção da segunda câmara, efetuada apenas pelas operárias. Espera-se que atue como um controle das estruturas a serem comparadas.

Tratamento 2 - Metade de fungo: número fixo de operárias (foi a média da população de ninhos de 6 meses), com a metade do peso médio do jardim de fungo $(7,25 \mathrm{~g})$ encontrado em colônias de 6 meses $(\mathrm{N}=5)$. Espera-se que as operárias confeccionem a estrutura de acordo com o volume do fungo, ou seja, dimensões reduzidas em relação ao controle.

Tratamento 3 - Dobro de fungo: número fixo de operárias (foi a média da população de ninhos de 6 meses), com o dobro do peso médio do jardim de fungo $(29,0 \mathrm{~g})$ encontrado em colônias de 6 meses $(\mathrm{N}=5)$. Espera-se que as operárias confeccionem a estrutura de acordo com o volume do fungo, ou seja, dimensões aumentadas ao dobro em relação ao controle.

Tratamento 4 - Sem fungo: número fixo de operárias (foi a média da população de ninhos de 6 meses), sem jardim de fungo ( $\mathrm{N}=5$ ). Espera-se que as operárias confeccionem independente da presença do jardim de fungo uma câmara, com dimensões similares ao controle, ou seja, uma testemunha experimental. 


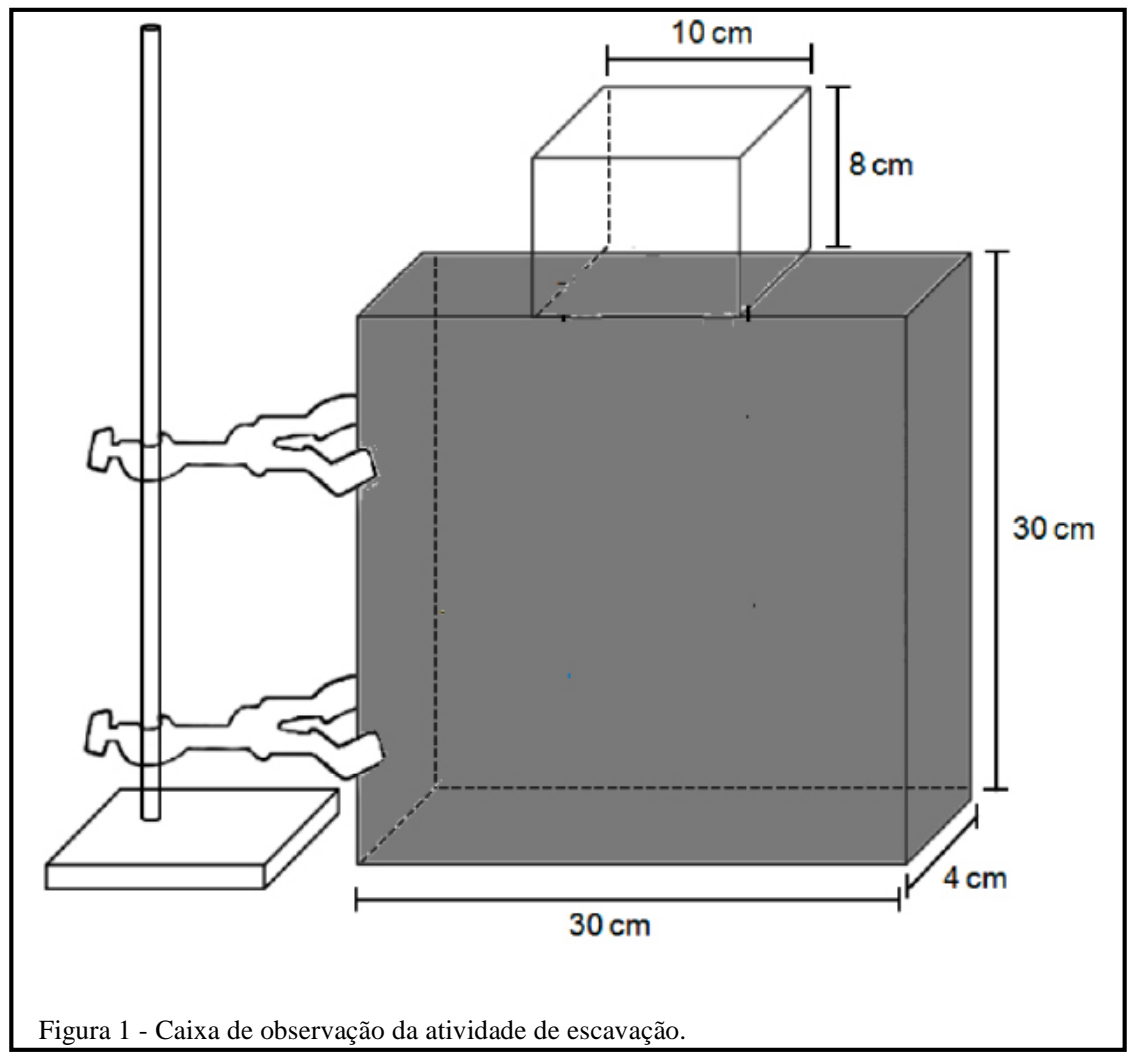

As variáveis estudadas foram:

a) parâmetros morfológicos das estruturas (tamanho e espessura dos túneis e câmara formada $(\mathrm{cm})$ );

b) fluxo das atividades das operárias na arena em que o jardim de fungo, prole, rainha e operárias foram dispostos. Foi filmado por 72 horas e amostrado 1 minuto a cada hora de filmagem. Sistema de filmagem por câmera e captação das imagens pelo software iuVCR, sendo amostrada 1 colônia a cada tratamento; c) volume de solo escavado a cada 24 horas, por pesagem (g). Peso úmido e seco, secagem por 3 dias a $70{ }^{\circ} \mathrm{C}$.

Espera-se que as operárias construam uma câmara para abrigar o jardim de fungo de acordo com o seu volume, dentro do tempo previsto. Corroborando a hipótese de que o jardim de fungo atua como um molde para a construção da câmara.

\section{Análises Estatísticas}

Os dados de morfologia das estruturas construídas pelas formigas foram submetidos a Análise de Variância (ANOVA) $(\alpha=0,05)$. O fluxo das atividades das operárias carregando pellet de solo e jardim de fungo foi submetido ao teste de KruskalWallis e, posteriormente, ao pós teste StudentNewman-Keuls $(\alpha=0,05)$. Os dados de volume de solo foram submetidos à Análise de Variância (ANOVA) $(\alpha=0,05)$, comparando-se as séries experimentais em cada intervalo de tempo. As análises estatísticas foram processadas pelo programa SigmaPlot 11.0.

\section{RESULTADOS}

O tratamento 4 (sem fungo) não apresentou nenhuma câmara, apenas túneis, em contraposição às demais séries experimentais que apresentaram no mínimo 2 câmaras. $O$ comprimento das câmaras foi de $9,7 \pm 5,2 \mathrm{~cm}$ para o padrão, $7,9 \pm 4,6 \mathrm{~cm}$ para metade de fungo, $9,3 \pm 3,9 \mathrm{~cm}$ para dobro de fungo (ANOVA, $\mathrm{F}_{3: 37}=13,370, \mathrm{P}<0,001$ ). A largura foi de $3,6 \pm 1,2 \mathrm{~cm}$ para o padrão, $4,0 \pm 0,0 \mathrm{~cm}$ para metade de fungo, $4,0 \pm 0,0 \mathrm{~cm}$ para dobro de fungo (ANOVA, $\left.\mathrm{F}_{3: 37}=96,862, \mathrm{P}<0,001\right)$. Finalmente, a altura foi de $3,3 \pm 1,9 \mathrm{~cm}$ para o padrão, $3,7 \pm 2,2 \mathrm{~cm}$ para metade de fungo, $3,9 \pm 2,0 \mathrm{~cm}$ para dobro de fungo (ANOVA, $\mathrm{F}_{3: 37}=10,965, \mathrm{P}<0,001$ ). O comprimento (tratamento 1 : $11,9 \pm 8,9 \mathrm{~cm}$; tratamento $2: 12,1 \pm 8,7 \mathrm{~cm}$; tratamento 3 : $11,5 \pm 5,2 \mathrm{~cm}$ e tratamento $4: 15,7 \pm 5,1 \mathrm{~cm})$ e espessura (tratamento 1: 1,9 $\pm 0,8 \mathrm{~cm}$; tratamento 2: $1,7 \pm 1,2 \mathrm{~cm}$; tratamento 3: $1,5 \pm 0,4 \mathrm{~cm}$ e tratamento $4: 2,09 \pm 1,1 \mathrm{~cm}$ ) dos túneis não diferiram estatisticamente entre as séries experimentais, sendo (ANOVA, $\mathrm{F}_{3.55}=0,829$, $\mathrm{P}=0,484) \quad$ e $\quad$ (ANOVA, $\quad \mathrm{F}_{3: 52}=0,925, \quad \mathrm{P}=0,436$ ), respectivamente. 
O fluxo das operárias carregando pellet de solo por minuto durante as 72 horas, diferiu estatisticamente entre as séries experimentais (Kruskal-Wallis, $\mathrm{H}=89,729$, d.f. $=3, \mathrm{P}<0,001$ ). O tratamento 1 que apresentava o mesmo volume de fungo ao encontrado nas colônias de 6 meses (Padrão), diferiu estatisticamente de todas as demais séries experimentais (Student-Newman-Keuls, $\mathrm{P}<0,05$ ), com fluxo mediano de 29 operárias por minuto a cada hora. O tratamento 2 (Metade de fungo) e tratamento 3 (Dobro de fungo) não diferiu estatisticamente, com fluxo mediano de operária de 21,7 e 22,6, respectivamente. O tratamento 4 (Sem fungo) diferiu de todos os demais, com fluxo mediano de 4,9 operária por minuto a cada hora. O fluxo das operárias carregando jardim de fungo e prole para o interior do ninho por minuto durante as 72 horas diferiu estatisticamente entre as séries experimentais (Kruskal-Wallis, $\mathrm{H}=29,004$, d.f. $=2, \mathrm{P}<0,001$ ).

$\mathrm{O}$ volume do solo escavado foi resultado da taxa de escavação das operárias. Interessantemente, nas primeiras 24 horas as operárias escavaram similarmente entre as 4 séries experimentais, não havendo diferenças estatísticas entre elas (Padrão: 123,6g; Metade de fungo:105,2g; Dobro de fungo: 99,9g e Sem fungo: 84,2g) (ANOVA, $\mathrm{F}_{3: 19}=2,661$, $\mathrm{P}=0,083)$. No intervalo de $24-48$ horas, houve diferença estatística entre as séries experimentais (Padrão: 147,8g; Metade de fungo: 124,8g; Dobro de fungo: 111,2g e Sem fungo: 18,8g) (ANOVA, $\left.\mathrm{F}_{3: 19}=14,799, \mathrm{P}<0,001\right)$. E o mesmo fato ocorreu no intervalo de 48-72 horas (Padrão: 121,6g; Metade de fungo: 105,4g; Dobro de fungo: $120,1 \mathrm{~g}$ e Sem fungo: 7,6g) (ANOVA, $\mathrm{F}_{3 ; 19}=14,799, \mathrm{P}<0,001$ ) (Figura 2).

\section{DISCUSSÃO}

Os resultados corroboram a hipótese de que o jardim de fungo atua como um molde para a construção da câmara. A ausência de uma estrutura funcional, tal como, uma câmara quando o jardim de fungo está ausente, comprova a hipótese. Além disso, as maiores taxas de escavação foram similares nas 24 primeiras horas, mas, no tratamento 4 (sem fungo), decresceu quando comparamos com as demais séries experimentais. Esse padrão de atividade de escavação e transporte de fungo, também foi observado em Acromyrmex lundi, com uma alta taxa de escavação no início do experimento, com uma redução ao decorrer do tempo (FRÖHLE \& ROCES, 2009) e também em outras espécies de formigas (RASSE \& DENEUBOURG, 2001; BUHL et al., 2005).
De acordo com BUHL et al. (2004), esse decréscimo na atividade no final da dinâmica de escavação pode ser explicado por dois possíveis mecanismos: o primeiro, corresponde à redução da atividade por meio da percepção de sinais específicos dos indivíduos, tais como a concentração de $\mathrm{CO}_{2}$ (COX \& BLANCHARD, 2000) ou taxa de encontros entre as formigas (GORDON, 1993). O segundo invoca as propriedades de propagação do processo de recrutamento, levando a atividade a estacionar de acordo com uma determinada densidade de formigas (DENEUBOURG \& FRANKS, 1995). Em nosso estudo, como havia uma população fixa, mas com uma variação no volume de fungo e prole, pode-se concluir que o decréscimo na atividade de escavação foi modulado pelo volume de jardim de fungo e prole acolhido em um ninho construído por elas. Não obstante, podemos descartar os mecanismos modeladores da escavação proposto por BUHL et al. (2004), pois os volumes de solo foram similares nas primeiras 24 horas entre as séries experimentais.

Outro resultado interessante é que, as estruturas formadas e volume de solo escavado não diferiram em relação aos diferentes volumes de jardim de fungo. Havíamos hipotetizado que o jardim de fungo atua como um molde tridimensional para o tamanho final da câmara, ou seja, de acordo com o seu crescimento, elas aumentam as suas dimensões, limitado pelas proporções do jardim de fungo, como discutido por FRÖHLE \& ROCES, 2009. Mas, como foi utilizado um número fixo de operárias por tempo fixo de escavação, a taxa foi similar em todas, independentemente do volume de fungo oferecido. Sabe-se que o tamanho do grupo é uma determinante importante para a taxa de escavação, pois se aumentar o tamanho diretamente aumenta-se a complexidade estrutural (BUHL et al., 2004). Entretanto, podese dizer que ocorreu uma compensação pela força operária entre o tratamento 3 (Metade de fungo) e 4 (Dobro de fungo), pois as operárias tiveram que carregar mais jardim de fungo para o interior do ninho no tratamento 4 , em relação ao tratamento 3 , reduzindo o número de operárias envolvidas na escavação. Por outro lado, o tratamento 3 carregou todo o jardim de fungo nas primeiras 24 horas, enquanto o tratamento 4 demorou 72 horas para carregar.

Adicionalmente, foi verificado que em todas as séries experimentais em que havia jardim de fungo, foram encontradas 2 câmaras de tamanhos similares. $\mathrm{O}$ número, bem como as dimensões das câmaras foram similares às encontradas em ambiente natural para a mesma espécie de estudo (CAMARGO et al., 2011), resultado da população fixa de operárias. 

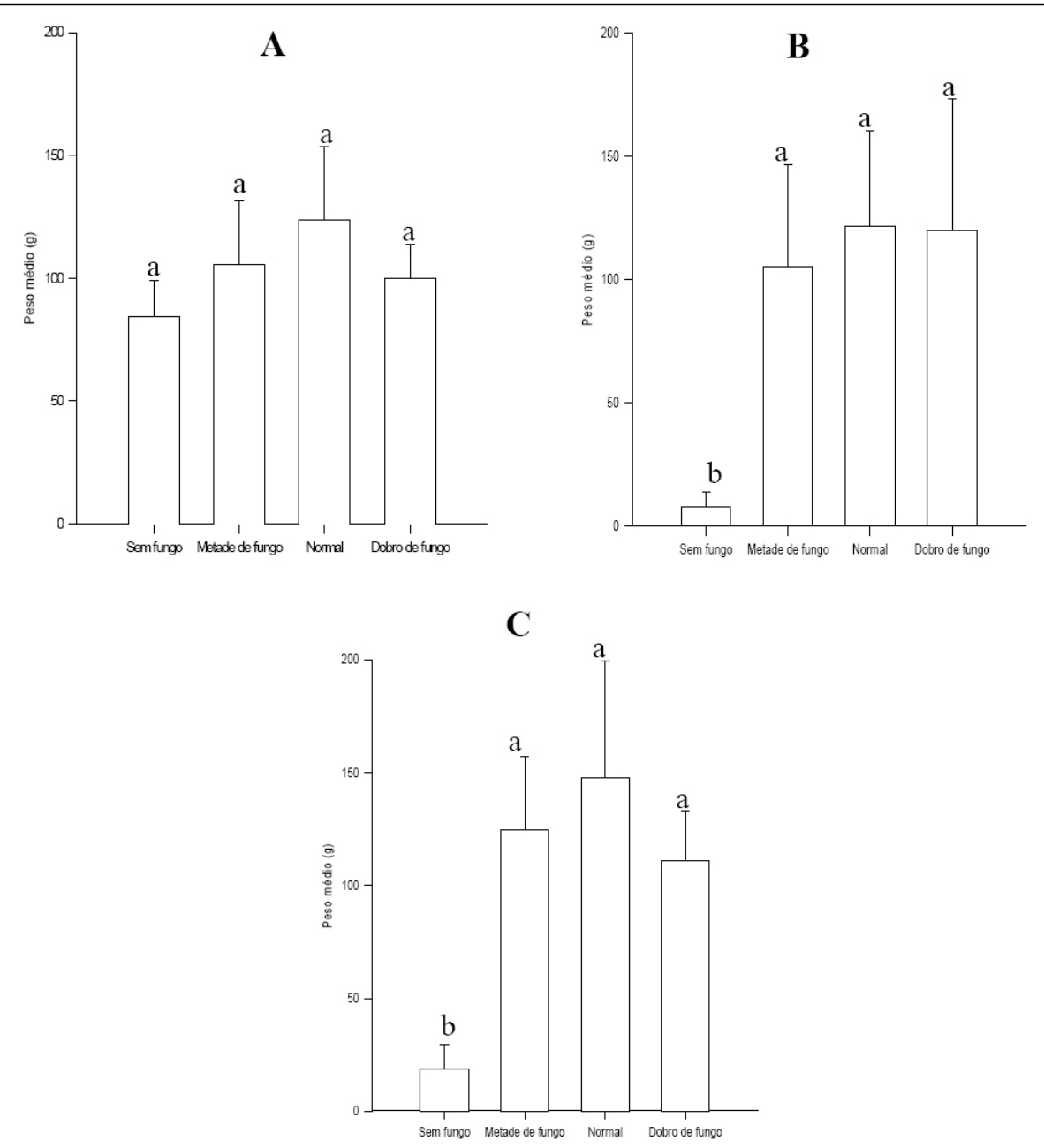

Figura 2 - Peso seco de solo escavado (g) de: A) 0-24 horas; B) 24-48 horas; C) 48-72 horas; Letras iguais indicam ausência de diferença significativa pela comparação pareada múltipla (Teste de Tukey, $\alpha=0,05$ ).

O presente estudo demonstrou experimentalmente que o jardim de fungo atua como um molde para a construção da câmara. Adicionalmente, podemos hipotetizar que a decisão para a construção de nova câmara em um ninho incipiente ocorre principalmente pelas operárias, as quais aumentam o ninho de acordo com o crescimento da colônia, promovendo a movimentação da rainha, jardim de fungo e prole para as câmaras, de acordo com a necessidade.

\section{AGRADECIMENTOS}

Gostaríamos de agradecer ao Sr. Francisco, pela coleta de solo, e ao Dr. Ricardo T. Fujihara, pela ajuda no sistema de filmagem. $\mathrm{O}$ primeiro autor agradece ao $\mathrm{CNPq}$, pela bolsa concedida pela modalidade Pós doutorado Junior.

\section{REFERÊNCIAS}

AUTUORI, M. Contribuição para o conhecimento da saúva (Atta spp - Hymenoptera- Formicidae). Arquivos do Instituto Biológico, v.13, p.137-50, 1942.

BRIAN, M.V. Social insects: ecology and behavioural biology. Chapman and Hall: New York, 1983.377 p.

BUHL, J. et al. Self-organized digging activity in ant colonies. Behavioral Ecology and Sociobiology, v. 58, p.9-17, 2005. Disponível em: <http://dx.doi:10.1007/s00265-004-0906-2>. Acesso em: 06 dez. 2012. doi: 10.1007/s00265-004-0906-2.

BUHL, J. et al. Nest excavation in ants: group size effects on the size and structure of tunneling networks. Naturwissenschaften, v.91, p.602-606, 2004. Disponível em: <http://cognition.ups-tlse. fr/_guyt/documents/articles/52.pdf>. Acesso em: 06 dez. 2012 doi: 10.1007/s00114-004-0577-x.

CAMARGO, R.S. et al. Digging effort in leaf-cutting ant queens (Atta sexdens rubropilosa) and its effects on survival and colony 
growth during the claustral phase. Insectes Sociaux, v.58, p.17-22, 2011. Disponível em: <http://dx. doi: 10.1007/s00040-010-01105>. Acesso em: 06 dez. 2012. doi: 10.1007/s00040-010-0110-5.

CASSIL, D. et al. Nest complexity, group size and brood rearing in the fire ant, Solenopsis invicta. Insectes Sociaux, v.49, p.158-163, 2002. Disponível em: 〈http://dx.doi: 10.1007/s00040-010-01105>. Acesso em: 06 dez. 2012. doi: 10.1007/s00040-010-0110-5 >.

CEUSTERS, R. Simulation du nid naturel des fourmis par des nids artificiels placés sur un gradient de température. Actes des Colloques Insestes Sociaux,v.3, p.235-241, 1986.

COX, M.D.; BLANCHARD, G.B. Gaseous templates in ant nests. Journal of Theorical Biology, v. 204, p. 223-238, 2000. Disponível em: <http://dx.doi.org/10.1006/jtbi.2000.2010>. Acesso em: 06 dez. 2012. doi: http://dx.doi.org/10.1006/jtbi.2000.2010.

DENEUBOURG, J.L.; FRANKS, N.R. Collective control without explicit coding: the case of communal nest excavation. Journal of Insect Behavior, v.4, p.417-432, 1995.

FRÖHLE, K.; ROCES, F. Underground agriculture: the control of nest size in fungus-growing ants. In: THERAULAZ, G.et al. (Eds.) From insect nest to human architecture. p.95-104, 2009.
GORDON, D.M. et al. What is the function of encounter patterns in ant colonies? Animal Behavior, v.45, 1083-1100, 1993.

HANSELL, M.H. Built by animals: the natural history of animal architecture. Oxford : Oxford University Press, 2007. 256p.

HÖLLDOBLER, B.; WILSON, E.O. The superorganism: the beauty, elegance and strangeness of insect societies. New York: W.W. Norton \& Company, 2009. 544p.

MOREIRA, A.A. et al. Nest architecture of Atta laevigata (F. Smith, 1858) (Hymenoptera: Formicidae). Studies on Neotropical Fauna and Environment, v.39, p.109-116, 2004. Disponível em: <http:// dx.doi.org/10.1080/01650520412331333756>. Acesso em: 06 dez. 2012. doi: http://dx.doi.org/10.1080/01650520412331333756.

RASSE, P.; DENEUBOURG, J.L. Dynamics of nest excavation and nest size regulation of Lasius niger (Hymenoptera: Formicidae). Journal of Insect Behavior, v.14, p.433-449, 2001. Disponível em: <http://www.springerlink.com/content/q8216362x0v2g0n0/>. Acesso em: 06 dez. 2012. doi: 10.1007/s00040-010-0110-5.

THERAULAZ, G. et al. The mechanisms and rules of coordinated building in social insects. In: Detrain C. et al. (Eds). Information Processing in Social Insects. Birkhäuser Verlag Basel/Switzerland, p. 309-330, 1999. 